\title{
Safety and metabolic advantages of steroid withdrawal after 6-months post-transplant in de novo kidney transplantation: 1-year prospective cohort study
}

\author{
Jun Bae Bang, Su Hyung Lee, Chang-Kwon Oh
}

Department of Surgery-Transplantation, Ajou University Hospital, Suwon, Korea

Background: This prospective multicenter study aimed at investigating the safety and metabolic advantages of steroid withdrawal therapy in kidney transplant recipients with tacrolimus-MMF based immunosuppression.

Methods: We analyzed 179 recipients who received kidney transplantation from March 2016 and September 2018. In 179 recipients, 114 patients maintained immunosuppressive regimen including steroid (SC group). The remaining 65 patients were determined to withdraw steroid therapy after 6 months post-transplant (SW group), who satisfied steroid withdrawal protocol consisted of stable serum creatinine, absence of rejection and no proteinuria. During study period, oral glucose tolerance tests (OGTTs) were performed every 3 month to evaluate the change of glucose metabolism.

Results: The estimated glomerular filtration rates at 12 months post-transplant were $67.29 \pm 20.29 \mathrm{~mL} / \mathrm{min} / 1.73 \mathrm{~m}^{2}$ in SC group and $73.72 \pm 17.57 \mathrm{~mL} / \mathrm{min} / 1.73 \mathrm{~m}^{2}$ in SW group $(\mathrm{P}<0.001)$. The acute rejection occurred to 15 recipients in SC group $(13.2 \%)$ and no acute rejection occurred to SW group recipients. After 6-months post-transplant, OGTT revealed that recipients in SW group was improved in glucose metabolism. Additionally, total cholesterol, high-density lipoprotein, low-density lipoprotein and blood pressure decreased after withdrawal of steroid in SW group.

Conclusions: Six-month withdrawal of steroid in recipients with low immunological risk and stable graft function can be safely conducted and result in improvement of metabolic profiles. The stable recipients without acute rejection and proteinuria can safely withdraw steroid out of maintenance immunosuppressive regimen after 6 months post-transplant. 